\title{
TWO CONJECTURES IN THE THEORY OF POINCARÉ DUALITY GROUPS
}

\author{
F. E. A. JOHNSON
}

\begin{abstract}
We show that it is not possible both to realise every Poincare Duality group as an aspherical manifold and to construct, for each Poincaré complex $X$, a Poincaré Duality group having the same integral homology as $X$.
\end{abstract}

In this note we wish to point out a relationship between two problems in the theory of Poincaré Duality groups, which, for dialectical purposes, we state as conjectures. They are

Homology Equivalence Conjecture. For any finite Poincaré complex $X$, not homotopy equivalent to $S^{2}$ or $\mathbf{R} P^{2}$, there is a finitely presented Poincaré Duality group $G$ and a homology equivalence $f: K(G, 1) \rightarrow X$.

Realisation Conjecture. If $G$ is a finitely presented Poincaré Duality group then there is a closed manifold $X_{G}$ of homotopy type $K(G, 1)$.

By a homology equivalence $f: Y \rightarrow X$ we mean a map which induces homology/cohomology isomorphisms with respect to all local coefficient systems on $X$. The Homology Equivalence Conjecture is the natural extension to Poincaré complexes of a problem first raised for smooth closed manifolds by Kan and Thurston in [5]. We shall prove

THEOREM 1. The Homology Equivalence and Realisation Conjectures are not both true.

We begin with

Proposition 2. If $f: X \rightarrow Y$ is a homology equivalence between connected spaces $X, Y$ which have the homotopy type of $\mathrm{CW}$ complexes, then, for any loopspace $B, f^{*}$ : $[Y, B] \rightarrow[X, B]$ is bijective.

Proof. The suspension of $f, S f: S X \rightarrow S Y$, induces isomorphisms in singular homology with simple integer coefficients, so, since $S X$ and $S Y$ are simply connected, is a homology equivalence. Hence, for any space $Z$, we have bijections

$$
(S f)^{*}:[S Y, Z] \stackrel{\simeq}{\rightarrow}[S X, Z] \text { and } f^{*}:[Y, \Omega Z] \stackrel{\simeq}{\rightarrow}[X, \Omega Z]
$$

The results follows on writing $B=\Omega Z$. Q.E.D.

Recall that to any finitely dominated Poincare complex, more generally to any space $X$ satisfying Poincaré Duality with integer coefficients (possibly twisted) we can associate a stable spherical fibration $\nu_{X}$, the Spivak fibration of $X$ [3], [7], which

Received by the editors March 3, 1980.

1980 Mathematics Subject Classification. Primary 20J10, 57B10; Secondary 57E30. 
plays the role of a generalised normal bundle. There is the following, first proved in this generality by Spivak, generalising an earlier result of Atiyah [1], [7].

Proposition 3 (Atryah-Spivak). Let $f: X \rightarrow Y$ be a homology equivalence between finite orientable Poincaré complexes. Then $f^{*}\left(\nu_{Y}\right)=\nu_{X}$.

Proof of Theorem 1. We shall show that if the Homology Equivalence Conjecture is true then the Realisation Conjecture is false.

Let $B G$ (resp. $B T O P$ ) be the classifying space for stable spherical fibrations (resp. stable topological microbundles). $B G$ and $B T O P$ are both loopspaces; in fact, they are infinite loopspaces [2]. Choose a simply connected Poincaré complex $X$ such that, if $\nu_{X}$ is classified by $c_{X} \in[X, B G]$, then $c_{X}$ does not belong to $\operatorname{Im} J$, $J:[X, B T O P] \rightarrow[X, B G]$. For example, as $X$ we may take the 5-dimensional Poincaré complex with $e_{1}(X) \neq 0$ constructed by Gitler and Stasheff in [4]. As mentioned in [4], the universal class $e_{1}$ was first introduced as the first obstruction to constructing a cross-section of the fibration $B P L \rightarrow B G, e_{1} \in H^{3}\left(B G ; Z_{2}\right) \simeq Z_{2}$ $\oplus \mathbf{Z}_{2}$. However, as is now well known, $e_{1}$ is the first obstruction to sectioning $B T O P \rightarrow B G$. This follows easily from the homotopy equivalence $T O P / P L \simeq$ $K\left(\mathbf{Z}_{2}, 3\right)$ of Kirby-Siebenmann [6]. Hence $c_{X}$ does not belong to the image of $J:[X, B T O P] \rightarrow[X, B G]$.

Now assume that the Homology Equivalence Conjecture is true, and apply it to produce a finitely presented Poincaré Duality group $H$ and a homology equivalence $f: K(H, 1) \rightarrow X$. If $K(H, 1)$ is not homotopy equivalent to a finite complex then, by a result of Kirby-Siebenmann [6], $K(H, 1)$ is not homotopy equivalent to a closed manifold, disproving the Realisation Conjecture. Hence suppose that $K(H, 1)$ is equivalent to a finite complex. By Proposition 2, we have a commutative square

$$
\begin{array}{ccc}
{[X, B T O P]} & \stackrel{f^{*}}{\leftrightarrows} & {[K(H, 1), B T O P]} \\
\downarrow J & & \downarrow J \\
{[X, B G]} & \stackrel{f^{*}}{\leftrightarrows} & {[K(H, 1), B G]}
\end{array}
$$

Now $H$ is certainly orientable, since $H_{1}(H, Z) \cong H_{1}(X, Z)=0$, so that, by Proposition $3, f^{*}\left(c_{X}\right)=c_{K(H, 1)}$. Since $c_{X}$ does not lift to BTOP, neither does $c_{K(H, 1)}$, and $K(H, 1)$ is not homotopy equivalent to any closed topological manifold. Q.E.D.

Of course, the possibility remains that both conjectures are false.

\section{REFERENCES}

1. M. F. Atiyah, Thom complexes, Proc. London. Math. Soc. 11 (1961), 291-310.

2. J. M. Boardman and R. M. Vogt, Homotopy everything H-spaces, Bull. Amer. Math. Soc. 74 (1968), 1117-1122.

3. W. Browder, Poincare spaces, their normal fibrations and surgery, Invent. Math. 17 (1972), 191-202.

4. S. Gitler and J. Stasheff, The first exotic class of BF, Topology 4 (1963), 257-266.

5. D. M. Kan and W. P. Thurston, Every connected space has the homology of a $K(\pi, 1)$, Topology 15 (1976), 253-258.

6. R. C. Kirby and L. C. Siebenmann, Foundational essays on topological manifolds, Ann. Math Studies, no. 88, Princeton Univ. Press, Princeton, N. J., 1977.

7. M. Spivak, Spaces satisfying Poincaré Duality, Topology 6 (1967), 77-101.

Department of Mathematics, University College London, London WCIE 6BT, England 